\title{
THREE-QUBIT ENTANGLEMENT WITNESSES WITH THE FULL SPANNING PROPERTIES
}

\author{
SEUNG-HYEOK KYE
}

\begin{abstract}
We construct three-qubit entanglement witnesses with the properties that all the partial transposes have the spanning properties. These witnesses determine faces for separable states whose interior lies in the interior of PPT states, and so they detect sets of PPT entangled states with nonempty interiors. These witnesses also detect all kinds of bi-separable pure product states. Our construction depends on the observation that multi-partite entanglement witnesses correspond to multi-linear maps which are positive.
\end{abstract}

\section{INTRODUCTION}

The separability problem is one of the most important research topics in the whole theory of entanglement, and the criterion with the positive partial transposes [4, 22] is quite strong for this purpose. If we denote by $\mathbb{S}$ and $\mathbb{T}$ the convex sets of all separable and PPT states, respectively, then the criterion tells us that the relation $\mathbb{S} \subset \mathbb{T}$ holds. These two convex sets coincide only for two-qubit and qubit-qutrit cases [4, 16, 27. In order to distinguish separable states among PPT states, we have to understand the boundary structures of the convex set $\mathbb{S}$. Some parts of boundary of $\mathbb{S}$ are determined by the boundary of $\mathbb{T}$, but some parts of the boundary are located in the interior of the convex set $\mathbb{T}$. The former case can be more easily understood than the latter, because the boundary structures of the convex set $\mathbb{T}$ itself can be described in terms of subspaces. See [8] and [13] for bi-partite and multi-partite cases, respectively. In this context, the latter cases should be important topics to be studied.

The boundary of $\mathbb{S}$ consists of exposed faces which are determined by entanglement witnesses. An entanglement witness $W$ determines a face of $\mathbb{S}$ whose interior lies in the interior of $\mathbb{T}$ if and only if the set of PPT entangled states detected by $W$ has a nonempty interior in $\mathbb{T}$ if and only if all the partial transposes of $W$ has the spanning properties. This is known for bi-partite cases [9, 20], and easily extended to multipartite cases. We will say that an entanglement witness $W$ has the full spanning property when $W$ has this property. Such witnesses have been constructed for bipartite cases. See [10, 11] for $3 \otimes 3$ cases, and [5, 6] for $4 \otimes 4$ cases. Very recently, $2 \otimes 4$ entanglement witnesses with the full spanning properties have been constructed in [14].

1991 Mathematics Subject Classification. 81P15, 15A30, 46L05.

partially supported by NRFK 2009-0083521. 
The main purpose of this note is to construct three-qubit entanglement witnesses with the full spanning properties. With these witnesses, it is easy to construct threequbit boundary separable states with full ranks, as it was requested in [2]. Such states have been constructed for the two-qutrit case [12], and studied [2] in detail. See also [14] for such states in $2 \otimes 4$ case. It turns out that our witnesses also detect all kinds of bi-separable pure product states.

It was shown in [17] that an $n$-partite entanglement witness corresponds to a linear map from the tensor product of the first $(n-1)$ systems into the last system, which sends separable states into positive matrices. We interpret this linear map as a multilinear map with $n-1$ variables whose linearization is a positive linear map with respect to the maximal tensor product of matrix algebras in the category of function systems [7, 15]. This makes easy to construct required witnesses and determine if they have the full spanning properties or not.

In the next section, we will discuss how entanglement witnesses correspond to positive multi-linear maps, and consider the full spanning properties of them which are multi-partite version of the bi-spanning property [9]. We also introduce the notion of completely positivity for multi-linear maps. We construct above mentioned three-qubit witnesses in Section 3 together with three-qubit boundary separable states with full ranks. We close this note to discuss other possible notions of positivity for bilinear maps.

The author is grateful to Kyung Hoon Han for useful discussion as well as providing the references [7] and [15].

\section{Positive multi-Linear maps as Entanglement Witnesses}

A state $\varrho$ on the Hilbert space $\mathcal{H}=\mathbb{C}^{d_{1}} \otimes \mathbb{C}^{d_{2}} \otimes \cdots \otimes \mathbb{C}^{d_{n}}$ is called separable if it is the convex combination

$$
\varrho:=\sum_{i \in I} p_{i}\left|\xi_{i}\right\rangle\left\langle\xi_{i}\right|
$$

of pure product states $\left|\xi_{i}\right\rangle\left\langle\xi_{i}\right|$ with

$$
\left|\xi_{i}\right\rangle=\left|\xi_{1 i}\right\rangle\left|\xi_{2 i}\right\rangle \cdots\left|\xi_{n i}\right\rangle \in \mathbb{C}^{d_{1}} \otimes \mathbb{C}^{d_{2}} \otimes \cdots \otimes \mathbb{C}^{d_{n}}, \quad i \in I
$$

Therefore, $\varrho$ is a $d \times d$ density matrix with the dimension $d=\prod_{j=1}^{n} d_{j}$ of the whole Hilbert space $\mathcal{H}$. On the other hand, a Hermitian matrix $W$ is said to be an entanglement witness if it is not positive (positive semi-definite) but satisfies the inequality

$$
\langle\xi|W| \xi\rangle \geq 0
$$

for every product vector $|\xi\rangle=\left|\xi_{1}\right\rangle\left|\xi_{2}\right\rangle \cdots\left|\xi_{n}\right\rangle$ in $\mathcal{H}$. This property is also called blockpositivity in bi-partite cases. See [24]. We have

$$
\langle\xi|W| \xi\rangle=\langle W, \mid \bar{\xi}\rangle\langle\bar{\xi} \mid\rangle
$$


with the usual bi-linear pairing $\langle A, B\rangle=\operatorname{Tr}\left(A^{\mathrm{t}} B\right)=\sum a_{i j} b_{i j}$ for $A=\left[a_{i j}\right]$ and $B=\left[b_{i j}\right]$ in $M_{d}$, where $A^{\mathrm{t}}$ denotes the transpose of $A$.

For a given $d \times d$ matrix $W$ with $d=\prod_{i=1}^{n} d_{i}$, we write

$$
\begin{aligned}
W & =\sum_{i_{1}, j_{1}}\left|i_{1}\right\rangle\left\langle j_{1}\right| \otimes W_{i_{1}, j_{1}} \in M_{d_{1}} \otimes M_{d_{2} d_{3} \cdots d_{n}} \\
& =\sum_{i_{1}, j_{1}} \sum_{i_{2}, j_{2}}\left|i_{1}\right\rangle\left\langle j_{1}|\otimes| i_{2}\right\rangle\left\langle j_{2}\right| \otimes W_{i_{1} i_{2}, j_{1} j_{2}} \in M_{d_{1}} \otimes M_{d_{2}} \otimes M_{d_{3} d_{4} \cdots d_{n}} \\
& =\cdots \\
& =\sum_{i_{1}, j_{1}, \ldots, i_{n-1}, j_{n-1}}\left|i_{1}\right\rangle\left\langle j_{1}|\otimes \cdots \otimes| i_{n-1}\right\rangle\left\langle j_{n-1}\right| \otimes W_{i_{1} \cdots i_{n-1}, j_{1} \cdots j_{n-1}}
\end{aligned}
$$

which belongs to $M_{d_{1}} \otimes \cdots \otimes M_{d_{n-1}} \otimes M_{d_{n}}$. We associate the multi-linear map $\phi_{W}$ from $M_{d_{1}} \times \cdots \times M_{d_{n-1}}$ into $M_{d_{n}}$ by

$$
\phi_{W}\left(\left|i_{1}\right\rangle\left\langle j_{1}|, \cdots,| i_{n-1}\right\rangle\left\langle j_{n-1}\right|\right)=W_{i_{1} \cdots i_{n-1}, j_{1} \cdots j_{n-1}} \in M_{d_{n}} .
$$

Conversely, for a multi-linear map $\phi$ from $M_{d_{1}} \times \cdots \times M_{d_{n-1}}$ into $M_{d_{n}}$, we associate a matrix $W_{\phi} \in M_{d}$ by

$$
W_{\phi}=\sum_{i_{1}, j_{1}, \ldots, i_{n-1}, j_{n-1}}\left|i_{1}\right\rangle\left\langle j_{1}|\otimes \cdots \otimes| i_{n-1}\right\rangle\left\langle j_{n-1}\right| \otimes \phi\left(\left|i_{1}\right\rangle\left\langle j_{1}|, \cdots,| i_{n-1}\right\rangle\left\langle j_{n-1}\right|\right) .
$$

The correspondences $\phi \mapsto W_{\phi}$ and $W \mapsto \phi_{W}$ are nothing but the Choi-Jamiołkowski isomorphisms [3, 18] for bi-partite case of $n=2$.

For a given multi-linear map $\phi$, we have

$$
\begin{aligned}
\left\langle\xi_{1}\right| \cdots\left\langle\xi_{n}\right| & W_{\phi}\left|\xi_{1}\right\rangle \cdots\left|\xi_{n}\right\rangle \\
& =\sum\left\langle\xi_{1} \mid i_{1}\right\rangle\left\langle j_{1} \mid \xi_{1}\right\rangle \cdots\left\langle\xi_{n-1} \mid i_{n-1}\right\rangle\left\langle j_{n-1} \mid \xi_{n-1}\right\rangle \cdot \\
& \left\langle\xi_{n}\left|\phi\left(\left|i_{1}\right\rangle\left\langle j_{1}|, \cdots,| i_{n-1}\right\rangle\left\langle j_{n-1}\right|\right)\right| \xi_{n}\right\rangle \\
& =\left\langle\xi_{n}\left|\phi\left(\left|\bar{\xi}_{1}\right\rangle\left\langle\bar{\xi}_{1}|, \cdots,| \bar{\xi}_{n-1}\right\rangle\left\langle\bar{\xi}_{n-1}\right|\right)\right| \xi_{n}\right\rangle,
\end{aligned}
$$

and so, we see that $W_{\phi}$ satisfies the condition (2) if and only if $\phi$ is positive, that is, $\phi\left(x_{1}, \ldots, x_{n-1}\right)$ is positive whenever all of $x_{1}, \ldots, x_{n-1}$ are positive. We denote by $\mathbb{P}$ the convex cone of all positive multi-linear maps. We note that a bi-linear map $\phi$ from the product $V_{1} \times V_{2}$ of functions systems into a function system $V_{3}$ is positive in this sense if and only if its linearization from $V_{1} \otimes_{\max } V_{2}$ into $V_{3}$ is a positive linear map, where $V_{1} \otimes_{\max } V_{2}$ is the the maximal tensor product of function systems. See [7, 15].

We define the bilinear pairing $\langle\varrho, \phi\rangle$ for $\varrho \in M_{d}$ and a $(n-1)$-linear map $\phi$ by

$$
\langle\varrho, \phi\rangle=\left\langle\varrho, W_{\phi}\right\rangle=\operatorname{Tr}\left(W_{\phi} \varrho^{\mathrm{t}}\right) \text {. }
$$

Then the above discussion tells us that $\phi \in \mathbb{P}$ if and only if $\langle\varrho, \phi\rangle \geq 0$ for every $\varrho \in \mathbb{S}$. By the separation theorem for a point outside of a closed convex set, we have the dual statement as follows:

Theorem 2.1. An $n$-partite state $\varrho$ is separable if and only if $\langle\varrho, \phi\rangle \geq 0$ for every positive $(n-1)$-linear map $\phi$. 
For a given positive $(n-1)$-linear map $\phi$, the set $\phi^{\prime}$ defined by

$$
\phi^{\prime}=\{\varrho \in \mathbb{S}:\langle\varrho, \phi\rangle=0\}
$$

is a face of the convex set $\mathbb{S}$. Extreme points of the convex set $\phi^{\prime}$ are determined by the set $P[\phi]$ of product vectors $|\xi\rangle=\left|\xi_{1}\right\rangle \cdot \cdots\left|\xi_{n}\right\rangle$ satisfying the relation

$$
\left\langle\xi\left|W_{\phi}\right| \xi\right\rangle=\left\langle W_{\phi}, \mid \bar{\xi}\right\rangle\langle\bar{\xi} \mid\rangle=\left\langle\xi_{n}\left|\phi\left(\left|\bar{\xi}_{1}\right\rangle\left\langle\bar{\xi}_{1}|, \cdots,| \bar{\xi}_{n-1}\right\rangle\left\langle\bar{\xi}_{n-1}\right|\right)\right| \xi_{n}\right\rangle=0 .
$$

We also consider the face $\phi^{\prime \prime}$ of $\mathbb{P}$ consisting of $\psi \in \mathbb{P}$ such that $\langle\varrho, \psi\rangle=0$ for each $\varrho \in \phi^{\prime}$. We note that $\phi^{\prime \prime}$ is the smallest exposed face of $\mathbb{P}$ containing $\phi$. Then we have the following:

$$
\psi \in \phi^{\prime \prime} \Longleftrightarrow\left\langle\xi\left|W_{\psi}\right| \xi\right\rangle=0 \quad \text { for each } \xi \in P[\phi] .
$$

We call a multi-linear map $\phi$ completely positive if $W_{\phi}$ is a positive matrix. This is the case if and only if the corresponding linear map from $M_{d_{1}} \otimes \cdots \otimes M_{d_{n-1}}$ into $M_{d_{n}}$ induced by $\phi$ is completely positive by [3]. From the above discussion, we have the following:

Proposition 2.2. For a given positive multi-linear map $\phi$, the following are equivalent:

(i) The set $P[\phi]$ of product vectors spans the whole space $\mathbb{C}^{d}$.

(ii) The smallest exposed face of $\mathbb{P}$ containing $\phi$ has no completely positive map.

We note that matrix algebras are nuclear as operator systems, and so the maximal tensor product and the minimal tensor product of matrix algebras coincide in the category of operator systems. Therefore, the above notion of complete positivity may be naturally applied for multi-linear maps in general operator systems whose linearization is completely positive with respect to the maximal tensor products. See [19], where the authors call those maps "jointly completely positive". See also [23] for discussion on related terminologies for operator spaces. It should be noted that the maximal tensor product of matrix algebras are different from the minimal tensor product in the category of function systems.

We say that $\phi$ (or the corresponding Hermitian matrix $W_{\phi}$ ) has the spanning property when it satisfies the above conditions in Proposition 2.2. The notion of spanning property for bi-partite case has been introduced in [21] in the context of optimality for entanglement witnesses. Our discussion above is nothing but the multi-partite analogue. It should be noted that even an indecomposable extreme positive map may not have the spanning property, as the Choi map shows. See [9].

Now, we turn our attention to the notion of partial transposes of multi-partite states. For a given subset $S$ of $\{1,2, \ldots, n\}$, we define the linear map $T(S)$ from $\bigotimes_{j=1}^{n} M_{d_{j}}$ into itself by

$$
\left(A_{1} \otimes A_{2} \otimes \cdots \otimes A_{n}\right)^{T(S)}:=B_{1} \otimes B_{2} \otimes \cdots \otimes B_{n}, \quad \text { with } B_{j}= \begin{cases}A_{j}^{\mathrm{t}}, & j \in S, \\ A_{j}, & j \notin S .\end{cases}
$$


A state $\varrho$ is said to be of PPT if $\varrho^{T(S)}$ is positive for every subset $S$. The convex set $\mathbb{T}$ of all PPT states is the intersection of convex sets

$$
\mathbb{T}^{S}=\left\{\varrho: \varrho^{T(S)} \text { is positive }\right\}
$$

through subsets $S$ of $\{1,2, \ldots, n\}$. Faces of $\mathbb{T}$ can be described in terms of subspaces [13]. Especially, a PPT state $\varrho$ is in the interior of $\mathbb{T}$ if and only if $\varrho^{T(S)}$ has full rank for each subset $S$.

For a product vector $|\xi\rangle=\left|\xi_{1}\right\rangle \otimes \cdots \otimes\left|\xi_{n}\right\rangle$, we also define the partial conjugate $|\xi\rangle^{\Gamma(S)}$ by

$$
\left(\left|\xi_{1}\right\rangle \otimes \cdots \otimes\left|\xi_{n}\right\rangle\right)^{\Gamma(S)}:=\left|\eta_{1}\right\rangle \otimes \cdots \otimes\left|\eta_{n}\right\rangle, \quad \text { with }\left|\eta_{j}\right\rangle= \begin{cases}\left|\bar{\xi}_{j}\right\rangle, & j \in S, \\ \left|\xi_{j}\right\rangle, & j \notin S,\end{cases}
$$

where $|\bar{\xi}\rangle$ denotes the conjugate of $|\xi\rangle$. We say that a positive multi-linear map $\phi$ has the full spanning property if $W_{\phi}^{T(S)}$ has the spanning property for every subset $S$. This is equivalent to the condition

$$
\operatorname{span}\left\{|\xi\rangle^{\Gamma(S)}: \xi \in P[\phi]\right\}=\mathcal{H}, \quad \text { for each subset } S \subset\{1,2, \ldots, n\} .
$$

We also say that $\phi$ is decomposable if it is in the convex hull of

$$
\left\{\phi \in \mathbb{P}: W_{\phi}^{T(S)} \text { is positive }\right\}
$$

through subsets $S$ of $\{1,2, \ldots, n\}$. It is clear that $\phi$ is decomposable if and only if $\langle\varrho, \phi\rangle \geq 0$ for every $\varrho \in \mathbb{T}$.

We are ready to state the main theorem in this section. The proof will be omitted, because it is simple and almost identical to the bi-partite cases, for which we refer to Chapter 8 of the survey note [20]. Especially, a map with the full spanning property must be indecomposable. See Figure 1 for the surrounding geometry of separable and PPT states.

Theorem 2.3. Let $\phi$ be a positive multi-linear map. Then the following are equivalent:

(i) $\phi$ has the full spanning property.

(ii) The interior of the face $\phi^{\prime}$ lies in the interior of $\mathbb{T}$.

(iii) The smallest exposed face $\phi^{\prime \prime}$ containing $\phi$ has no decomposable map.

(iv) The set $\{\varrho \in \mathbb{T}:\langle\varrho, \phi\rangle<0\}$ of PPT entangled states detected by $\phi$ has a nonempty interior in $\mathbb{T}$.

\section{Construction for the three-qubit CASE}

We begin with the following bilinear map $\phi$ from $M_{2} \times M_{2}$ into $M_{2}$ which sends $\left(\left[x_{i j}\right],\left[y_{i j}\right]\right) \in M_{2} \times M_{2}$ to

$$
\left(\begin{array}{cc}
s x_{22} y_{11} & x_{12} y_{12}-x_{12} y_{21}+x_{21} y_{12}+x_{21} y_{21} \\
x_{12} y_{12}+x_{12} y_{21}-x_{21} y_{12}+x_{21} y_{21} & t x_{11} y_{22}
\end{array}\right),
$$




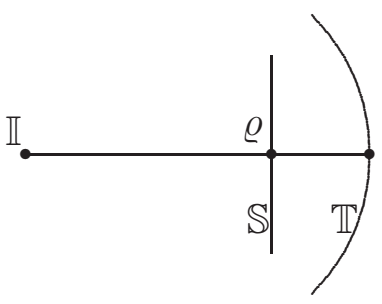

FiguRE 1. The vertical line segment represents the face $\phi^{\prime}$ of $\mathbb{S}$ determined by the positive map $\phi$ with the full spanning property. The points $\mathbb{I}$ and $\varrho$ represent an interior point of $\mathbb{S}$ and a boundary separable state with full ranks, respectively. The line segment from $\mathbb{I}$ to $\varrho$ can be extended within the convex set $\mathbb{T}$, because $\varrho$ is an interior point of $\mathbb{T}$. The curve represents the boundary of the convex set $\mathbb{T}$.

where $s, t$ are positive numbers with the relation $s t=8$. The corresponding entanglement witness is given by

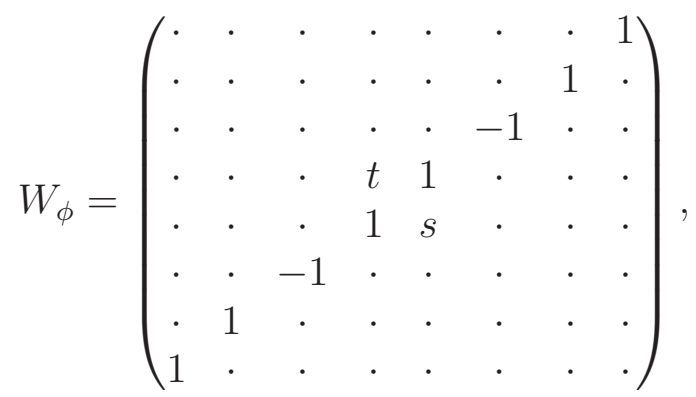

where $\cdot$ denotes zero.

In order to show that $\phi$ is a positive bilinear map, we consider the rank one projection $P_{\alpha}=\left(\begin{array}{cc}1 & \bar{\alpha} \\ \alpha & |\alpha|^{2}\end{array}\right)$. We see that $\phi$ sends $\left(P_{\alpha}, P_{\beta}\right)$ to

$$
\left(\begin{array}{cc}
s|\alpha|^{2} & \alpha \beta-\bar{\alpha} \beta+\alpha \bar{\beta}+\bar{\alpha} \bar{\beta} \\
\alpha \beta+\bar{\alpha} \beta-\alpha \bar{\beta}+\bar{\alpha} \bar{\beta} & t|\beta|^{2}
\end{array}\right)
$$

whose determinant is given by the nonnegative number

$$
D=|\alpha \beta-\bar{\alpha} \bar{\beta}|^{2}+|\alpha \bar{\beta}+\bar{\alpha} \beta|^{2} .
$$

We proceed to determine the product vectors in $P[\phi]$. First of all, we look at the image under $\phi$ when one of variables is $P_{0}=|0\rangle\langle 0|$ or $P_{\infty}=|1\rangle\langle 1|$ as follows:

$$
\begin{array}{ll}
\phi(|0\rangle\langle 0|, y)=\left(\begin{array}{cc}
0 & 0 \\
0 & t y_{22}
\end{array}\right), & \phi(|1\rangle\langle 1|, y)=\left(\begin{array}{cc}
s y_{11} & 0 \\
0 & 0
\end{array}\right) \\
\phi(x,|0\rangle\langle 0|)=\left(\begin{array}{cc}
s x_{22} & 0 \\
0 & 0
\end{array}\right), \quad \phi(x,|1\rangle\langle 1|)=\left(\begin{array}{cc}
0 & 0 \\
0 & t x_{11}
\end{array}\right) .
\end{array}
$$

We see that

$$
\begin{aligned}
\langle\zeta|\phi(|0\rangle\langle 0|, y)| \zeta\rangle=0 & \Longleftrightarrow|\zeta\rangle=|0\rangle \text { or } y=|0\rangle\langle 0| \\
\langle\zeta|\phi(|1\rangle\langle 1|, y)| \zeta\rangle=0 & \Longleftrightarrow|\zeta\rangle=|1\rangle \text { or } y=|1\rangle\langle 1| \\
\langle\zeta|\phi(x,|0\rangle\langle 0|)| \zeta\rangle=0 & \Longleftrightarrow|\zeta\rangle=|1\rangle \text { or } x=|0\rangle\langle 0| \\
\langle\zeta|\phi(x,|1\rangle\langle 1|)| \zeta\rangle=0 & \Longleftrightarrow|\zeta\rangle=|0\rangle \text { or } x=|1\rangle\langle 1| .
\end{aligned}
$$


From these, we found the following product vectors in $P[\phi]$ :

$$
|\xi\rangle 0\rangle 1\rangle, \quad|\xi\rangle 1\rangle 0\rangle, \quad|0\rangle \eta\rangle 0\rangle, \quad|1\rangle \eta\rangle 1\rangle, \quad|0\rangle 0\rangle \zeta\rangle, \quad|1\rangle 1\rangle \zeta\rangle,
$$

with arbitrary $|\xi\rangle,|\eta\rangle,|\zeta\rangle \in \mathbb{C}^{2}$. We note that product vectors in (7) span the 6dimensional subspace of $\mathbb{C}^{2} \otimes \mathbb{C}^{2} \otimes \mathbb{C}^{2}$ whose orthogonal complement is spanned by $|0\rangle 1\rangle 1\rangle$ and $|1\rangle 0\rangle 0\rangle$. We also note that these product vectors are parameterized by six Riemann spheres. If we put these six Riemann spheres in a suitable cyclic way then two adjacent Riemann spheres have exactly one common point. See Figure 2.

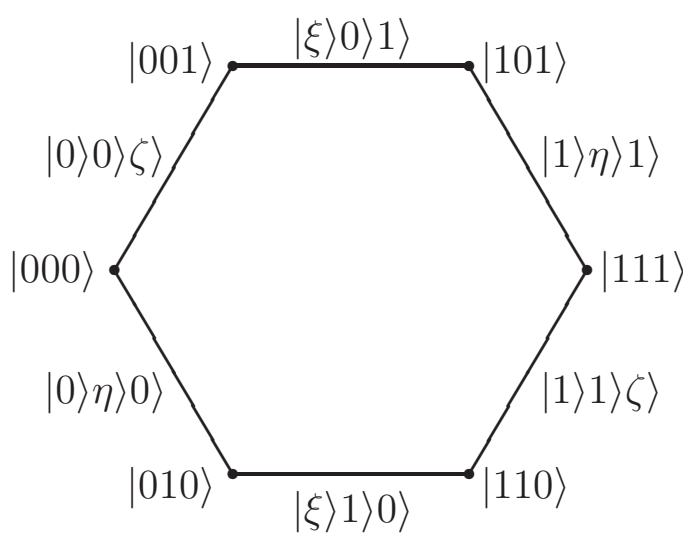

FiguRE 2. Each line segment represents the Riemann sphere which parameterizes product vectors with a single variable.

To find further product vectors in $P[\phi]$, we solve the equation $D=0$ with (6) to get the nonzero solutions $(\alpha, \beta)$ :

$$
\left(a_{1} \omega, b_{1} \omega^{7}\right), \quad\left(a_{2} \omega^{3}, b_{2} \omega^{5}\right), \quad\left(a_{3} \omega^{5}, b_{3} \omega^{3}\right), \quad\left(a_{4} \omega^{7}, b_{4} \omega\right),
$$

where $a_{i}, b_{i}$ are arbitrary positive numbers and $\omega=e^{\frac{\pi i}{4}}$ is the 8 -th root of unity. The corresponding $2 \times 2$ matrices $\phi\left(P_{\alpha}, P_{\beta}\right)$ are given by

$$
\begin{array}{ll}
\left(\begin{array}{cc}
s a_{1}^{2} & r a_{1} b_{1} \omega \\
r a_{1} b_{1} \omega^{7} & t b_{1}^{2}
\end{array}\right), & \left(\begin{array}{cc}
s a_{2}^{2} & r a_{2} b_{2} \omega^{7} \\
r a_{2} b_{2} \omega & t b_{2}
\end{array}\right), \\
\left(\begin{array}{cc}
s a_{3}^{2} & r a_{3} b_{3} \omega \\
r a_{3} b_{3} \omega^{7} & t b_{3}
\end{array}\right), & \left(\begin{array}{cc}
s a_{4}^{2} & r a_{4} b_{4} \omega^{7} \\
r a_{4} b_{4} \omega & t b_{4}^{2}
\end{array}\right),
\end{array}
$$

where $r=2 \sqrt{2}$. The corresponding kernel vectors are also given by

$$
\left(r b_{1}, s a_{1} \omega^{3}\right)^{\mathrm{t}}, \quad\left(r b_{2}, s a_{2} \omega^{5}\right)^{\mathrm{t}}, \quad\left(r b_{3}, s a_{3} \omega^{3}\right)^{\mathrm{t}}, \quad\left(r b_{4}, s a_{4} \omega^{5}\right)^{\mathrm{t}} .
$$

By (3), we have the following product vectors in $P[\phi]$ :

$$
\begin{gathered}
\zeta_{1}\left(a_{1}, b_{1}\right)=\left(1, a_{1} \omega^{7}\right)^{\mathrm{t}} \otimes\left(1, b_{1} \omega\right)^{\mathrm{t}} \otimes\left(b_{1}, u a_{1} \omega^{3}\right)^{\mathrm{t}} \\
\zeta_{2}\left(a_{2}, b_{2}\right)=\left(1, a_{2} \omega^{5}\right)^{\mathrm{t}} \otimes\left(1, b_{2} \omega^{3}\right)^{\mathrm{t}} \otimes\left(b_{2}, u a_{2} \omega^{5}\right)^{\mathrm{t}} \\
\zeta_{3}\left(a_{3}, b_{3}\right)=\left(1, a_{3} \omega^{3}\right)^{\mathrm{t}} \otimes\left(1, b_{3} \omega^{5}\right)^{\mathrm{t}} \otimes\left(b_{3}, u a_{3} \omega^{3}\right)^{\mathrm{t}} \\
\zeta_{4}\left(a_{4}, b_{4}\right)=\left(1, a_{4} \omega\right)^{\mathrm{t}} \otimes\left(1, b_{4} \omega^{7}\right)^{\mathrm{t}} \otimes\left(b_{4}, u a_{4} \omega^{5}\right)^{\mathrm{t}} \\
7
\end{gathered}
$$


where $u=\frac{s}{r}=\frac{s}{2 \sqrt{2}}$. Therefore, the set $P[\phi]$ consists of product vectors in (77) and (8). It is trivial to check the condition (44) for the full spanning property of $\phi$.

Theorem 3.1. For any positive numbers $s, t$ with st $=8$, the $\operatorname{map} \phi: M_{2} \times M_{2} \rightarrow M_{2}$ defined by (5) is a positive bilinear map with the full spanning property.

Typical PPT entangled states detected by $\phi$ are so called $X$-shaped states [1, 25, 26, For example, we consider the following state

$$
\varrho=\left(\begin{array}{cccccccc}
1 & \cdot & \cdot & \cdot & \cdot & \cdot & \cdot & -1 \\
\cdot & 1 & \cdot & \cdot & \cdot & \cdot & -1 & \cdot \\
\cdot & \cdot & 1 & \cdot & \cdot & 1 & \cdot & \cdot \\
\cdot & \cdot & \cdot & s / 2 \sqrt{2} & -1 & \cdot & \cdot & \cdot \\
\cdot & \cdot & \cdot & -1 & t / 2 \sqrt{2} & \cdot & \cdot & \cdot \\
\cdot & \cdot & 1 & \cdot & \cdot & 1 & \cdot & \cdot \\
\cdot & -1 & \cdot & \cdot & \cdot & \cdot & 1 & \cdot \\
-1 & \cdot & \cdot & \cdot & \cdot & \cdot & \cdot & 1
\end{array}\right)
$$

It is clear that $\varrho$ is of PPT whenever $s t=8$, but we have

$$
\langle\varrho, \phi\rangle=\frac{s t}{2 \sqrt{2}}+\frac{s t}{2 \sqrt{2}}-8=\frac{8}{\sqrt{2}}-8<0,
$$

as it is required.

In order to look for bi-separable states detected by $\phi$, we consider

$$
\begin{aligned}
& \xi_{1}(\alpha)=(1, \bar{\alpha})_{A}^{\mathrm{t}} \otimes(0,1, \alpha, 0)_{B C}^{\mathrm{t}}=\left(0,1, \alpha, 0,0, \bar{\alpha},|\alpha|^{2}, 0\right)^{\mathrm{t}} \\
& \xi_{2}(\alpha)=(1, \bar{\alpha})_{B}^{\mathrm{t}} \otimes(1,0,0, \alpha)_{A C}^{\mathrm{t}}=\left(1,0, \bar{\alpha}, 0,0, \alpha, 0,|\alpha|^{2}\right)^{\mathrm{t}} \\
& \xi_{3}(\alpha)=(1, \bar{\alpha})_{C}^{\mathrm{t}} \otimes(1,0,0, \alpha)_{A B}^{\mathrm{t}}=\left(1, \bar{\alpha}, 0,0,0,0, \alpha,|\alpha|^{2}\right)^{\mathrm{t}}
\end{aligned}
$$

Then we see that

$$
\left\langle W_{\phi}, \mid \xi_{i}(\alpha)\right\rangle\left\langle\xi_{i}(\alpha) \mid\right\rangle=-2|\alpha|^{2} \pm\left(\alpha^{2}+\bar{\alpha}^{2}\right)<0
$$

for each $i=1,2,3$, whenever $\alpha^{2} \in \mathbb{C} \backslash \mathbb{R}$.

We proceed to search boundary separable states with full ranks. For simplicity, we fix $s=t=2 \sqrt{2}$ in the definition of $\phi$. We note that the following ten product vectors

$$
|000\rangle, \quad|001\rangle, \quad|010\rangle, \quad|101\rangle, \quad|110\rangle, \quad|111\rangle, \quad \zeta_{i}(1,1), \quad i=1,2,3,4
$$

in $P[\phi]$ span the whole space. All kinds of partial conjugates of them also span the whole space, and so any nontrivial convex combination of the ten corresponding pure product states must be boundary separable states with full ranks. For more concrete 
examples, we denote by $\varrho_{0}$ the separable states given by first six product vectors:

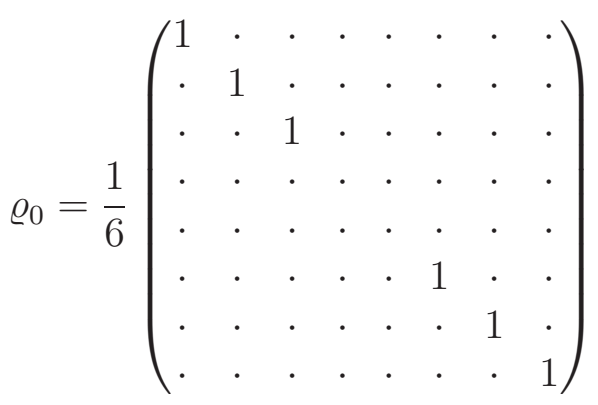

We also denote by $\varrho_{1}$ the separable state given by the last four product vectors:

$$
\varrho_{1}=\frac{1}{8 \sqrt{2}}\left(\begin{array}{cccccccc}
\sqrt{2} & -1 & \cdot & \cdot & \cdot & \cdot & \sqrt{2} & -1 \\
-1 & \sqrt{2} & \cdot & \cdot & \cdot & \cdot & -1 & \sqrt{2} \\
\cdot & \cdot & \sqrt{2} & -1 & \cdot & 1 & \cdot & \cdot \\
\cdot & \cdot & -1 & \sqrt{2} & -1 & \cdot & \cdot & \cdot \\
\cdot & \cdot & \cdot & -1 & \sqrt{2} & -1 & \cdot & \cdot \\
\cdot & \cdot & 1 & \cdot & -1 & \sqrt{2} & \cdot & \cdot \\
\sqrt{2} & -1 & \cdot & \cdot & \cdot & \cdot & \sqrt{2} & -1 \\
-1 & \sqrt{2} & . & . & . & \cdot & -1 & \sqrt{2}
\end{array}\right)
$$

Then we see that

$$
\varrho_{\lambda}=(1-\lambda) \varrho_{0}+\lambda \varrho_{1}
$$

is a boundary separable state with full ranks whenever $0<\lambda<1$.

\section{Discussion}

In this note, we have considered two notions of positivity for multi-linear maps, whose linearizations satisfy natural positivity with respect to the maximal tensor products in the categories of functions systems and operator systems, respectively.

We may consider various other notions for positivity of multi-linear maps. We list up some candidates for bilinear maps $\phi: M_{A} \times M_{B} \rightarrow M_{C}$ :

- $y \mapsto \phi(x, y): M_{B} \rightarrow M_{C}$ is completely positive for each positive $x \in M_{A}$.

- $x \mapsto \phi(x, y): M_{A} \rightarrow M_{C}$ is completely positive for each positive $y \in M_{B}$.

- The corresponding linear map $M_{A} \otimes M_{B} \rightarrow M_{C}$ is positive.

The above properties have obvious dual objects in the contexts of bi-separable states [1]. For example, $\phi$ satisfies the first condition if and only if the corresponding linear map $M_{A} \rightarrow M_{B} \otimes M_{C}$ is positive if and only if $\langle\varrho, \phi\rangle \geq 0$ for each $A$ - $B C$ bi-separable states $\varrho$. Therefore, a tri-partite entanglement witness detects an entangled state which is not bi-separable if and only if the corresponding bilinear map satisfies the above three properties simultaneously. It would be interesting to formulate the above properties in the framework of general function systems and/or operator systems. 


\section{REFERENCES}

[1] A. Acin, D. Bruß, M Lewenstein and A. sanpera, Classification of Mixed Three-Qubit States, Phys. Rev Lett. 87 (2001), 040401.

[2] L. Chen and D. Ž. Djoković, Boundary of the set of separable states, preprint. arXiv:1404.0738.

[3] M.-D. Choi, Completely positive linear maps on complex matrices, Linear Alg. Appl. 10 (1975), $285-290$.

[4] M.-D. Choi, Positive linear maps, Operator Algebras and Applications (Kingston, 1980), pp. 583-590, Proc. Sympos. Pure Math. Vol 38. Part 2, Amer. Math. Soc., 1982.

[5] D. Chruściński and G. Sarbicki, Exposed positive maps: a sufficient condition, J. Phys. A: Math. Theor. 45 (2012), 115304.

[6] D. Chruściński and G. Sarbicki, Exposed positive maps in $M_{4}(\mathbb{C})$, Open Sys. Inf. Dyn. 19 (2012), 1250017.

[7] E. G. Effros, Injectives and tensor products for convex sets and $C^{*}$-algebras, Lecture Note, NATO Institute on "Facial strusture of compact convex sets" Swansea, 1972.

[8] K.-C. Ha and S.-H. Kye, Construction of entangled states with positive partial transposes based on indecomposable positive linear maps, Phys. Lett. A 325 (2004), 315-323.

[9] K.-C. Ha and S.-H. Kye, Optimality for indecomposable entanglement witnesses, Phys. Rev. A 86 (2012), 034301.

[10] K.-C. Ha and S.-H. Kye, Entanglement witnesses arising from Choi type positive linear maps, J. Phys. A: Math. Theor. 45 (2012), 415305.

[11] K.-C. Ha and S.-H. Kye, Exposedness of Choi type entanglement witnesses and applications to lengths of separable states, Open Syst. Inf. Dyn. 20 (2013), 1350012.

[12] K.-C. Ha and S.-H. Kye, Separable states with unique decompositions, Commun. Math. Phys. 328 (2014), 131-153.

[13] K.-C. Ha and S.-H. Kye, Multi-partite separable states with unique decompositions and construction of three-qubit entanglement with positive partial transpose, J. Phys. A: Math. Theor. 48 (2015), 045303.

[14] K.-C. Ha and S.-H. Kye, Construction of exposed indecomposable positive linear maps between matrix algebras, preprint, arXiv:1410.5545.

[15] K. H. Han, Tensor products of function systems revisited, preprint.

[16] M. Horodecki, P. Horodecki and R. Horodecki, Separability of mixed states: necessary and sufficient conditions, Phys. Lett. A 223 (1996), 1-8.

[17] M. Horodecki, P. Horodecki and R. Horodecki, Separability of n-particle mixed states: necessary and sufficient conditions in terms of linear maps, Phys. Lett. A 283 (2001), 1-7.

[18] A. Jamiołkowski, An effective method of investigation of positive maps on the set of positive definite operators, Rep. Math. Phys. 5 (1974), 415-424.

[19] A. Kavruk, V. I. Paulsen, I. G. Todorov and M. Tomforde, Tensor products of operator systems, J. Funct. Anal. 261 (2011), 267-299.

[20] S.-H. Kye, Facial structures for various notions of positivity and applications to the theory of entanglement, Rev. Math. Phys. 25 (2013), 1330002.

[21] M. Lewenstein, B. Kraus, J. Cirac and P. Horodecki, Optimization of entanglement witness, Phys. Rev. A 62 (2000), 052310.

[22] A. Peres, Separability Criterion for Density Matrices, Phys. Rev. Lett. 77 (1996), 1413-1415.

[23] G. Pisier and D. Shlyakhtenko, Grothendiecks theorem for operator spaces, Invent. Math. 150 (2002), 185-217.

[24] Ł. Skowronek, E. Størmer, and K. Zyczkowski, Cones of positive maps and their duality relations, J. Math. Phys. 50 (2009), 062106.

[25] S. Vinjanampathy and A. R. P. Rau, Generalized $X$ states of $N$ qubits and their symmetries, Phys. Rev. A 82 (2010), 032336.

[26] Y. S. Weinstein, Entanglement dynamics in three-qubit X states, Phys. Rev. A 82 (2010), 032326.

[27] S. L. Woronowicz, Positive maps of low dimensional matrix algebras, Rep. Math. Phys. 10 (1976), $165-183$.

Department of Mathematics and Institute of Mathematics, Seoul National UniVERsity, SEOUl 151-742, KoreA 